\title{
Elementos para la evaluación eficaz de productos naturales con posibles efectos antihipertensivos
}

\author{
Mario Francisco Guerrero \\ Departamento de Farmacia, Facultad de Ciencias, Universidad Nacional de Colombia, \\ Bogotá, D.C., Colombia
}

\begin{abstract}
Desde el contexto de la farmacología experimental, en este ensayo se discuten algunos criterios que pueden ser útiles para estimar el potencial de productos naturales con posible actividad farmacológica para el tratamiento de la hipertensión, una de las enfermedades de mayor impacto epidemiológico en Colombia y en el mundo. Se acude para ello al análisis de algunas pruebas farmacológicas accesibles en nuestro medio: modelos de hipertensión arterial en la rata de laboratorio, pruebas in vitro e in vivo, y análisis microscópicos, que implementados con los protocolos adecuados y cotejadas en conjunto, pueden arrojar información valiosa a la hora de considerar las perspectivas de un producto natural en el campo de la hipertensión arterial.
\end{abstract}

Palabras clave: evaluación de medicamentos, productos biológicos, hipertensión, resistencia vascular, ratas, modelos cardiovasculares.

\begin{abstract}
Elements for the effective evaluation of natural products with possible antihypertensive effects

Within the context of experimental pharmacology, some criteria are discussed for estimating the potential of natural products with possible pharmacological activity for the treatment of hypertension. This disorder has great epidemiological impact in Colombia as well as elsewhere. Discovery of these products can be accomplished by use of accessible pharmacological tests such as the following: developing models of arterial hypertension in the laboratory rat, in vitro tests, in vivo tests and microscopic analysis. Suitable protocols associated with these techniques can capture valuable information about possible effects on hypertension during the evaluation phase of a natural product.
\end{abstract}

Key words: drug evaluation, biological products, hypertension, vascular resistance, rats; models, cardiovascular.

La hipertensión arterial sigue siendo, pese a los importantes avances logrados en el conocimiento de su fisiopatología y de su abordaje terapéutico, uno de los trastornos que aún conlleva mayor morbimortalidad en el mundo (1). En Colombia, ella y los trastornos cardiovasculares asociados constituyen la

\footnotetext{
Correspondencia:

Mario Francisco Guerrero, Departamento de Farmacia, Facultad de Ciencias, Universidad Nacional de Colombia, Carrera 30 No. 45-03, edificio 450, oficina 311, apartado aéreo 14490, Bogotá, D.C., Colombia.

Teléfono: (571) 316 5000, extensión 14631, y 617 7462; fax: (571) 3165060

mfguerrerop@unal.edu.co
}

Recibido: 30/01/09; aceptado:13/04/09 primera causa de morbimortalidad, por encima de la violencia (2). Las medidas no farmacológicas, tales como el control de peso, la restricción en el consumo de sodio, la moderación en el consumo de alcohol, el ejercicio y el control del estrés, son definitivamente necesarias para prevenir o atenuar este trastorno, no obstante lo cual, la mayoría de pacientes requieren medicación adicional (3). Se dispone de una variedad de fármacos que utilizados de manera adecuada, de acuerdo con las características particulares de cada paciente, logran modificar el curso de la enfermedad (4). Sin embargo, un importante número de pacientes permanece vulnerable a las complicaciones de la hipertensión arterial y a los efectos adversos de la medicación $(5,6)$. 
Las alternativas farmacológicas a partir de productos naturales podrían contribuir a reducir el impacto de esta enfermedad. Todavía, más de la mitad de medicamentos disponibles se basan en productos naturales, porcentaje que se incrementa cuando se trata de aquéllos destinados a trastornos cardiovasculares (7). Su enorme diversidad química, la disponibilidad creciente de técnicas de aislamiento e identificación y la introducción de ensayos farmacológicos que permiten detectar moléculas potencialmente promisorias en un margen de tiempo razonable, son argumentos adicionales en favor de la vigencia de los productos naturales como fuente terapéutica $(8,9)$.

Colombia, dada su riquísima biodiversidad, posee una ventaja estratégica en este campo. La ley ambiental consagra no sólo la protección y el uso sostenible de los recursos naturales del país, sino, también, la investigación de la flora colombiana como un asunto prioritario en la asignación de recursos públicos (10). Pese al importante papel que los productos naturales, particularmente las plantas medicinales, han tenido en la medicina tradicional, sigue siendo escasa la investigación científica de sus propiedades farmacológicas, entre otros campos, en el de la hipertensión arterial. No obstante, esfuerzos recientes desde la academia abocados al estudio de especies con esos usos etnofarmacológicos, entre ellas: Salvia palifolia, o mastranto $(11,12)$, Croton schiedeanus, o almizclillo $(13,14)$, Calea prunifolia, o carrasposa (15) y Solanum tuberosum, o papa (16), podrían alentar el impulso de la investigación en esta área.

Los productos naturales han sido un campo fértil como fuente para la obtención de agentes antihipertensivos. Los inhibidores de la enzima convertidora de angiotensina, los bloqueadores del receptor beta y los antagonistas de angiotensina II, aunque obtenidos por síntesis, son ejemplos de fármacos que deben buena parte de su descubrimiento a un farmacóforo de origen natural (7). A su vez, extractos naturales pueden tener cabida en el manejo de pacientes hipertensos. Allium sativum, o ajo, es quizá el ejemplo más conocido (17).
En nuestro medio se dispone de pruebas farmacológicas que permiten estimar el potencial farmacológico de los productos naturales. Existen pruebas tanto in vitro como in vivo: in vitro, por sus inobjetables ventajas: la reducción en el número de animales de laboratorio, el control de los factores hemodinámicos que complican la identificación de los mecanismos de acción de una sustancia y la cuantificación precisa de parámetros de eficacia y potencia farmacológica; in vivo, porque si bien el principio de las tres eres, reemplazo, refinamiento y reducción, debe guiar la investigación farmacológica, es indispensable la preparación íntegra para evaluar los efectos cardiovasculares ejercidos por un compuesto dado.

Se requiere de un enfoque farmacológico global en el estudio de productos naturales como alternativa a las técnicas de tamización de alta eficiencia que copan la atención de centros abocados al descubrimiento y desarrollo de nuevos fármacos, cuyo esquema, dirigido a la identificación de moléculas con actividad sobre blancos específicos, deja de lado la posibilidad de identificar nuevos agentes farmacológicos con nuevos mecanismos de acción y, pese a la sofisticada tecnología que exige, adolece de una visión reduccionista que soslaya la naturaleza integrada y multifactorial de la enfermedad. La hipertensión arterial, como tantos otros, es un trastorno de etiología en esencia aún desconocida, cuyas alteraciones fisiopatológicas, cabe considerar, no se han abordado farmacológicamente en su totalidad.

Aunque es comprensible que el enfoque predominante en la investigación farmacológica del momento sea de tipo molecular, por el auge de la tamización de alta eficiencia, la química combinatoria y el modelado computacional de fármacos, no por ello el enfoque tradicional, que estudia el uso etnofarmacológico de los productos naturales, pierde vigencia. No es necesariamente el juego de derivados moleculares sobre un receptor el camino garantizado para encontrar alternativas terapéuticas más efectivas y con menores efectos adversos. La Organización Mundial de la Salud ha reconocido la medicina tradicional como un gran bloque de conocimiento 
para la atención primaria en salud (18), pero ésta necesita ser examinada libre del manto de ocultismo y magia al que suele ir ligada su práctica.

El rigor con que debe ser abordado, exige un margen de escepticismo con respecto al uso etnofarmacológico de plantas medicinales, particularmente aquéllas a las que se les atribuyen efectos antihipertensivos. La hipertensión arterial es en esencia una enfermedad asintomática, "la enemiga silenciosa". Requiere para su diagnóstico la medición controlada de esa variable hemodinámica que debe sobrepasar los "valores normales" en forma sostenida. Sólo cuando se producen las lesiones de los órganos diana de la hipertensión: corazón, cerebro, riñón, grandes vasos, retina, se presentan síntomas, por lo demás inespecíficos de la hipertensión arterial.

¿Cómo, entonces, las comunidades descubrieron el valor terapéutico de plantas medicinales con efectos antihipertensivos? Además, aunque inestimable, el uso tradicional no tiene por qué ser el único criterio que guíe la investigación en la validación farmacológica de las plantas medicinales. Disponiendo de técnicas eficientes de evaluación farmacológica una tamización "no etnofarmacológica" puede dar con extractos o compuestos promisorios.

No se trata tampoco de oponer a la farmacología de síntesis la etnofarmacología, ni a ésta, un enfoque farmacológico "no tradicional". Se trata dearmonizarlas. Aunqueelexamendelaactividad farmacológica puede llevar a la conclusión de que un extracto estandarizado es una opción adecuada como alternativa terapéutica cuando se identifica que son varios los principios activos que interactúan sinérgicamente para generar el efecto farmacológico global de una especie, una aproximación farmacológica más general tiene mucho que aportar a la farmacología de síntesis a la hora de buscar derivados farmacológicos de metabolitos de origen natural potencialmente promisorios.

\section{Evaluación del efecto sobre la presión arterial}

Una vez seleccionados los extractos, fracciones o compuestos de origen natural por evaluar, aspecto en lo que tienen especial importancia especies con usos etnofarmacológicos como hipotensoras y antihipertensivas, es necesario utilizar un modelo in vivo adecuado.

En un contexto farmacológico, el modelo debe ser lo más reproducible posible; es decir, debe permitir predecir razonablemente que un compuesto dado, independientemente de los mecanismos que ejerza, producirá efectos en el humano. La rata normotensa, por ejemplo, la rata Wistar, tan ampliamente utilizada en la tamizaciónfarmacológica de productos naturales, no es el modelo más conveniente sencillamente porque es un modelo de "normotensión", no de hipertensión.

Por eso, la ausencia de efectos sobre la presión arterial en la rata normotensa no debe ser motivo para descartar una fracción o un compuesto. Con respuestas variables, fármacos utilizados en clínica, incluyendo a bloqueadores del receptor de angiotensina, inhibidores de la enzima convertidora de angiotensina, bloqueadores del receptor beta, bloqueadores de canales de calcio, diuréticos y antihipertensivos de acción central, ejercen efectos notorios en ratas hipertensas, en tanto que en ratas normotensas son mucho menores (19-26). Es necesario acudir a modelos de hipertensión.

Posiblemente, el modelo in vivo más utilizado en farmacología experimental es el de la rata espontáneamente hipertensa (27). Sin embargo, aunque es muy sensible para detectar el efecto de fármacos como inhibidores de la enzima convertidora de angiotensina, antagonistas de angiotensina II, antagonistas de calcio o "vasodilatadores directos", no lo es tanto para bloqueadores del receptor beta y mucho menos para diuréticos y antogonistas de endotelina $(27,28)$.

Además, tiene un patrón genético de transmisión mendeliana, aspecto en el que difiere de la hipertensión esencial en el humano que es de naturaleza poligénica. Su sensibilidad farmacológica se explica en buena parte porque es un modelo que cursa con renina alta, en contraste con los modelos de renina baja, como los de rata Dahl o DOCA, cuya hipertensión está 
asociada a sobrecarga de volumen. Este aspecto tiene relación con lo hallado en la clínica: que los pacientes con renina alta son más sensibles al efecto de inhibidores de la enzima convertidora de angiotensina o antagonistas de angiotensina II, en tanto que los pacientes con renina baja, que tienden a tener sobrecarga de volumen, responden mejor a los diuréticos (29).

Una alternativa a la rata espontáneamente hipertensa, por su menor costo, es el de rata con hipertensión experimental por deficiencia de óxido nítrico, como la inducida con el agente L-NAME (N(G)-nitro-L-arginina-metiléster), inhibidor no selectivo de la enzima óxido nítrico sintetasa (30). Con el reconocimiento de la importancia del óxido nítrico en la fisiopatología de la hipertensión arterial, este modelo cobra mayor vigencia, no sólo para demostrar los efectos sobre la presión arterial, sino también para examinar las complicaciones de los órganos blanco (31,32). Su sensibilidad para detectar la actividad antihipertensiva supera a la rata espontáneamente hipertensa, dado que permite identificar, además de fármacos que actúan sobre el sistema renina-angiotensina-aldosterona, agentes que actúan sobre el sistema nervioso autónomo y diuréticos $(33,34)$.

Independientemente del modelo in vivo seleccionado, detalles importantes por tener en cuenta en el examen de los efectos antihipertensivos de extractos y productos naturales, son la duración del tratamiento, la dosis y la vía de administración. Haciendo un parangón con la clínica, así como se espera unas cuatro semanas para observar los efectos de la farmacoterapia en un paciente dado, también es necesario en una rata con hipertensión experimental esperar el tiempo suficiente, cuando menos, unas tres semanas, para examinar si el producto administrado resiste los ajustes hemodinámicos de la rata hipertensa (35).

Es frecuente que los productos naturales ejerzan efectos farmacológicos breves, aspecto que no sorprende considerando que los organismos vegetales y animales han tenido todo el tiempo de la evolución para sus interacciones mutuas.
Si los procesos de biotransformación determinan efectos breves en el extracto, es improbable que éste tenga futuro como fuente farmacológica. De ahí la importancia de demostrar efectos sostenidos en el tiempo.

Por otro lado, no cabe esperar mayores efectos farmacológicos de productos naturales con dosis excesivamente altas en la rata de laboratorio, próximas a un gramo por kilo, aun considerando que la tasa metabólica del roedor es muy superior a la del humano; a no ser por sus potenciales perspectivas como suplementos nutricionales, lo que, desde luego, tampoco carece de importancia. Además, tratándose de extractos y fracciones como posibles fuentes terapéuticas para un trastorno crónico, es clara la importancia de demostrar efectos por vía oral. En cualquier caso, esta aproximación farmacológica no es incompatible con aquélla orientada a obtener compuestos por síntesis o semisíntesis.

No basta con demostrar los efectos sobre la presión arterial, es importante determinar en una preparación in vivo los posibles mecanismos cardiovasculares inducidos por una sustancia. Una técnica útil para este propósito es la preparación de rata sin médula, que permite exponer una sustancia en estudio ante agonistas, antagonistas y mensajeros conocidos, hacer aproximaciones a su posible mecanismo de acción y confirmar los hallazgos farmacológicos de investigaciones in vitro, como las obtenidas con preparaciones vasculares.

Tiene la ventaja de permitir que los procesos de biotransformación entren en juego mientras se controlan los ajustes homeostáticos del sistema nervioso autónomo que, de estar presentes, complicarían la comprensión del efecto cardiovascular. Así, se pone a prueba en una preparación in vivo la aproximación farmacodinámica derivada de resultados in vitro. Es, por consiguiente, una herramienta muy útil en el estudio farmacológico de un compuesto; ayuda a discriminar interacciones sobre blancos farmacológicos clave en la fisiopatología de la hipertensión, entre otros: receptores beta (36), alfa (37), canales de calcio (38) y angiotensina $(39,40)$. 


\section{Evaluación del efecto vasodilatador}

Puesto que los parámetros que determinan la presión arterial son el gasto cardiaco y la resistencia vascular periférica, es necesario examinar sobre cuál de ellos actúa primariamente una sustancia antihipertensiva. Cierto que sea el uno o el otro, terminará incidiendo necesariamente en el otro, porque el organismo tiende a mantener el flujo sanguíneo tisular constante; sin embargo, dado que el evento fisiopatogénico central en la hipertensión esencial es el incremento sostenido de la resistencia vascular periférica, es importante identificar si un agente farmacológico afecta primariamente la resistencia vascular, porque esto significaría que sus efectos están más dirigidos hacia el centro fisiopatológico del trastorno.

Es claro que la elevación sostenida del tono del músculo liso vascular es el principal factor que determina el incremento de la resistencia, así que todo agente capaz de producir efectos vasodilatadores, podría ser útil como fuente farmacológica para la hipertensión. Por consiguiente, el estudio del efecto farmacológico sobre la resistencia vascular periférica tiene particular importancia en la búsqueda de agentes farmacológicos para la hipertensión. $\mathrm{Si}$ se examina con detalle, cabe considerar que aun fármacos que ejercen efectos directos sobre la precarga, como los diuréticos, o sobre la contractilidad miocárdica, como los bloqueadores del receptor beta, podrían atenuar primariamente la resistencia vascular periférica; es decir, atenuar la poscarga, no necesariamente como fenómeno exclusivamente debido a la disminución del gasto cardiaco $(41,42)$.

Una de las preparaciones in vitro especialmente útiles para examinar efectos vasodilatadores es la de anillos aislados de aorta. Como las técnicas de su tipo, posee entre sus ventajas: requerir pequeñas cantidades de sustancia, estar libre de los ajustes homeostáticos sistémicos que dificultan el estudio farmacodinámico de una sustancia y permitir la obtención con bastante precisión de parámetros clave en farmacología experimental, como concentración inhibitoria 50 $\left(\mathrm{Cl}_{50}\right)$ y efecto máximo $\left(\mathrm{E}_{\max }\right)$, indicadores de la potencia y eficacia de un fármaco. De hecho, los estudios de mecánica in vitro con esta preparación arrojan constantes de actividad muy similares a las que se describen en los estudios con radioligandos (43).

Una objeción que se hace a esta preparación es la de que corresponde a un vaso de conductancia, habida cuenta de que las alteraciones que conducen a la hipertensión arterial tienen lugar en vasos de resistencia. No obstante, hay que considerar que la túnica media de la aorta de la rata está compuesta fundamentalmente por músculo liso, a diferencia de la túnica media de la aorta del humano, que está formada por tejido elástico. Esta característica le permite a esta preparación responder eficazmente a los agentes que actúan sobre el músculo liso vascular. De hecho, un muy diverso grupo de fármacos vasodilatadores útiles en clínica ha demostrado actividad en los ensayos con anillos de aorta, entre otros, bloqueadores del receptor alfa (44), bloqueadores de canales de calcio $(45,46)$, bloqueadores del receptor beta $(47)$, mecanismos dependientes 0 independientes del endotelio vascular $(48,49)$, inhibidores de la enzima convertidora de angiotensina (50) y antagonistas de angiotensina II (51).

Dado que requiere apenas pequeñas cantidades de sustancia, esta preparación es muy útil para hacer el seguimiento bioguiado de un extracto con efectos vasodilatadores y para determinar, una vez aislados e identificados sus principales metabolitos, si éstos desempeñan efectos sinérgicos, hallazgo por cierto frecuente con productos naturales.

Una guía útil que orienta el fraccionamiento y ayuda a discriminar los posibles mecanismos de acción de un compuesto natural, es examinar los valores de potencia y eficacia vasodilatadora ante diferentes agentes vasoconstrictores. Sin duda, un compuesto tendrá interés si es capaz de producir vasodilatación completa $\left(\mathrm{E}_{\max }\right.$ $100 \%$ ) con $\mathrm{Cl}_{50}$ inferior al orden micromolar. No obstante, no existe un protocolo único que le permita al investigador determinar si tal o cual sustancia ejerce efectos vasodilatadores, por lo que es necesario enfrentarla ante varios agentes 
vasoconstrictores. Sería un error, por ejemplo, descartar un producto natural que carece o presenta poca actividad cuando se expone ante fenilefrina y $\mathrm{KCl}$ en la tamización farmacológica, porque con ello apenas se descartarían mecanismos de tipo alfa-adrenérgico o antagonismo de calcio, cuando cabe plantear otras posibilidades, como interacciones con angiotensina, entre tantas otras.

Entre las vías metabólicas conducentes a la vasodilatación que este tipo de preparaciones ha facilitado estudiar, se destacan las relacionadas con la generación de óxido nítrico (NO), el factor de relajación derivado del endotelio (52), mediador que, además de otros efectos fisiológicos, posee actividad antiagregante plaquetaria, natriurética y antiarterioesclerótica; de ahí su interés como blanco farmacológico en el tratamiento de la hipertensión y sus trastornos asociados (53-55).

Es abundante la literatura sobre productos naturales, compuestos flavonoides entre tantos, cuyos mecanismos están ligados a la producción de óxido nítrico. ¿Tiene, entonces, futuro farmacológico como agente antihipertensivo un producto natural que induzca la liberación de óxido nítrico o que incremente su actividad? Hay que considerar el papel dual que este mediador desempeña en los procesos fisiológicos. Mientras se mantenga la producción endotelial (constitutiva) de óxido nítrico, se producirán sus efectos vasodilatadores y antiagregantes, pero un exceso en la producción de este mediador llevará al incremento de generación de radicales tóxicos para la célula, como el peroxinitrito, producto de su reacción con anión superóxido (56). Es posible, entonces, que al pretender mejorar la actividad vasodilatadora de un compuesto natural que induzca la liberación de óxido nítrico, se produzca un efecto paradójico.

No obstante, el efecto sostenido aunque moderado en la inducción de la producción de óxido nítrico por un compuesto natural puede significar la protección a largo plazo sobre la integridad del endotelio vascular y del endocardio. El beneficio de la dieta rica en polifenoides, por ejemplo, se debe, al menos en parte, a mecanismos de este tipo $(57,58)$. Pero esto ubicaría al compuesto natural en el plano nutracéutico, no en el farmacológico, es decir, como suplemento y profiláctico, no como alternativa terapéutica.

Es necesario abordar, también, los posibles efectos farmacológicos que pueda ejercer una sustancia sobre el volumen sanguíneo, por la importancia que este componente tiene sobre el volumen de eyección, el gasto cardiaco y la presión arterial; de ahí el valor de los agentes diuréticos. Estos fármacos han resistido la prueba del tiempo en la terapia de la hipertensión arterial $(4,59)$. La arterioesclerosis progresiva de los vasos, especialmente de la aorta, es per se un factor de riesgo determinante en la morbimortalidad asociada a la hipertensión sistólica. Los diuréticos proporcionan alivio a la tensión generada sobre las paredes de la aorta endurecida, mejorando el pronóstico del paciente.

La evaluación de los efectos diuréticos en farmacología experimental con los cambios electrolíticos asociados, sodio y potasio particularmente, es sencilla, puede hacerse incluso en la rata normotensa (35). Es abundante, también, la información etnofarmacológica sobre productos naturales con posibles efectos diuréticos, por lo que su abordaje como terapéutica potencial para la hipertensión merece ser estudiado más en profundidad (60).

Además, hay que examinar los posibles efectos farmacológicos sobre la contractilidad y el cronotropismo del miocardio, para lo cual son preparaciones adecuadas, entre otras, las de aurícula derecha e izquierda, respectivamente (35). Permiten, entre sus aplicaciones, estudiar in vitro el efecto de agentes antagonistas de calcio y de bloqueadores del receptor beta sobre la frecuencia y el inotropismo cardíacos $(61,62)$. Conviene también, cotejar estos hallazgos con los obtenidos en preparaciones aisladas de útero, tráquea y conducto deferente que, aunque son órganos diferentes de corazón y vasos, ayudan a determinar el perfil farmacológico cardiovascular $(35,63)$.

En términos generales, existe una razonable correlaciónfarmacodinámicaentrepreparaciones 
in vitro e in vivo encaminadas a estudiar los parámetros hemodinámicos fundamentales en la presión arterial: resistencia vascular, contractilidad y frecuencia cardíaca. Así como diverso tipo de agentes vasodilatadores ejercen efectos en la preparación de anillos aislados de aorta, igual cosa ocurre con la preparación del "tren vascular posterior de rata", preparación íntegra en la que se mantiene el flujo sanguíneo constante mientras se examinan los efectos sobre la resistencia vascular $(64,65)$. Cosa similar ocurre entre las preparaciones íntegras con catéteres in situ acoplados a transductores de flujo sanguíneo, presión y frecuencia cardiaca, y las preparaciones aisladas de aurícula derecha e izquierda, cuando se trata de examinar los efectos sobre el inotropismo y el cronotropismo cardiacos $(66,67)$. Si ello no es así, hay que considerar la posibilidad de estar frente a profármacos, cuando no son los agentes que se administran los responsables del efecto, sino aquéllos en que se convierten, luego de que sufren biotransformación. En tal caso, es improbable que tengan efecto en las preparaciones in vitro.

En consecuencia, un flujograma de tamización para el abordaje de productos naturales con posible actividad antihipertensiva, debería contemplar tanto pruebas in vivo como pruebas in vitro, dado que son técnicas complementarias, examinando en uno y otro caso, los parámetros mencionados: resistencia vascular, contractilidad y frecuencia cardíaca, y teniendo en cuenta, además, que es indispensable que el agente demuestre efectos en un modelo crónico o subcrónico de hipertensión (figura 1).

\section{Evaluación del efecto sobre el remodelado cardiovascular}

En la hipertensión arterial el incremento sostenido del tono del músculo liso vascular genera una sobrecarga sobre las paredes del miocardio que, cuando sobrepasa el límite fisiológico, se traduce en una hipertrofia anómala, disfuncional, tanto del miocardio como del músculo liso vascular, fenómeno conocido como remodelado. Se presenta, además, proliferación de fibroblastos, con su consecuente excesiva producción de matriz colágena intersticial y acumulación de células inflamatorias tales como linfocitos y macrófagos.

Como resultado, se afecta progresivamente la capacidad contráctil del miocardio y la capacidad autorreguladora y dilatadora del

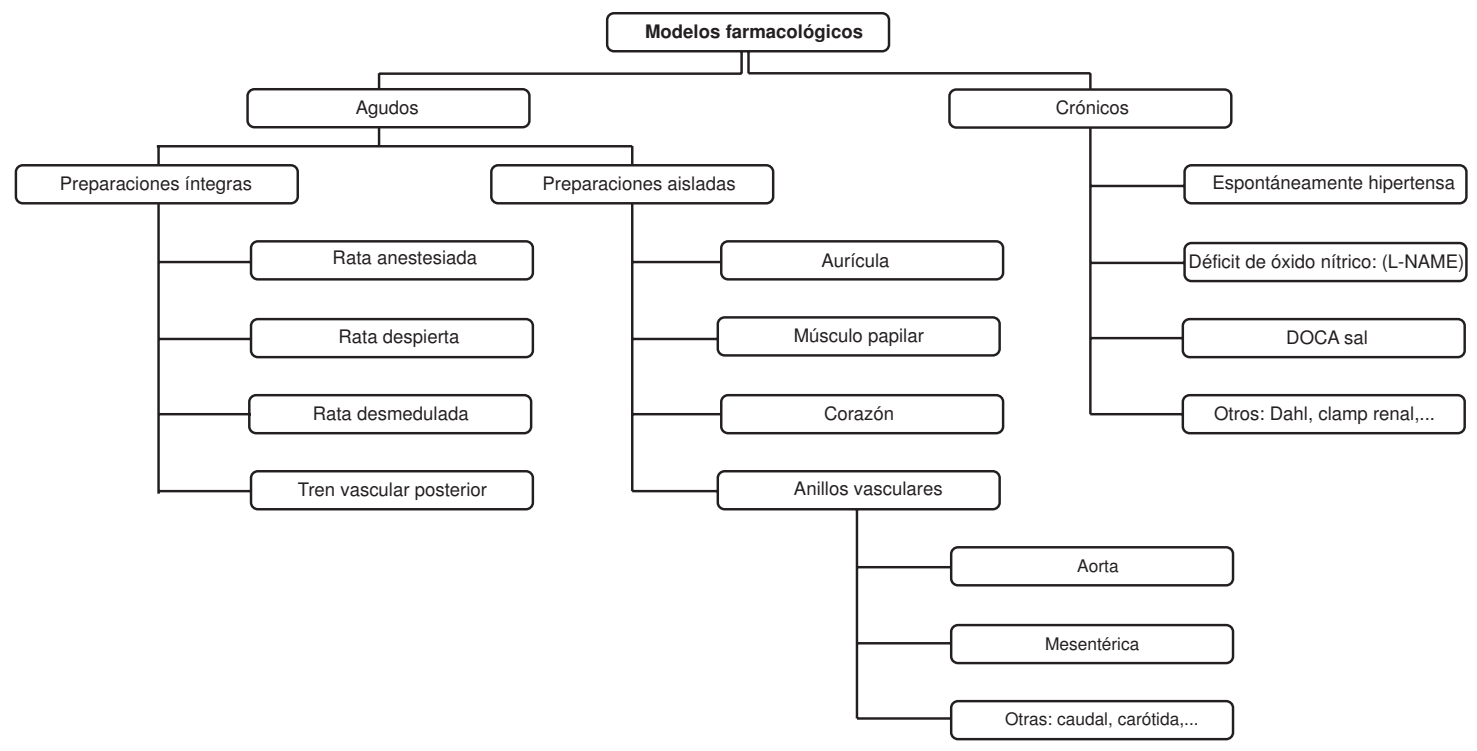

Figura 1. Modelos experimentales en rata de laboratorio para la evaluación de productos naturales con posibles efectos antihipertensivos. 
vaso (68). Además, el proceso de remodelado en los vasos coronarios conlleva un déficit de irrigación sanguínea para el corazón. Éste es otro componente de disfunción coronaria que, además, se agrava por la hipertrofia ventricular, dado que el notorio incremento en la tensión de las paredes ventriculares comprime el lecho vascular coronario. Se establece así un círculo vicioso que, si no se detiene, torna vulnerable el miocardio a accidentes coronarios y termina lesionando la fibra miocárdica, lo que se traduce en falla cardíaca.

Tanto el sistema renina-angiotensina-aldosterona como el sistema nervioso autónomo simpático, incrementan el remodelado $(69,70)$; de allí que los fármacos que bloquean uno y otro, como inhibidores de la enzima convertidora de angiotensina, antagonistas de angiotensina II, antagonistas de aldosterona y bloqueadores del receptor beta, no sólo ejercen efectos antihipertensivos sino que ejercen efectos antiremodelado. Esto explica, en buena parte, la utilidad especial de este tipo de fármacos en pacientes hipertensos con falla cardíaca, enfermedad coronaria o ambas. La identificación de compuestos naturales que interfieren esas vías metabólicas o aquéllas cuya investigación muestre que alivian la tensión del miocardio, suscitarán un particular interés, no sólo en el campo de la hipertensión arterial, sino en el de la terapia de isquemia miocárdica y la falla cardiaca.

Desde el punto de vista de remodelado cardiovascular, y por paradójico que en principio pueda parecer, el mantenimiento de los valores de presión dentro de "límites normales" no garantiza necesariamente que se detendrá la lesión sobre el miocardio y, por ende, que se reducirá el riesgo de enfermedad coronaria y falla cardiaca. Todo aquello que favorezca la tensión sobre las paredes del miocardio, favorece el remodelado. Si la terapia antihipertensiva dispara la respuesta del sistema nervioso autónomo simpático, del sistema renina-angiotensina-aldosterona 0 de ambos, las complicaciones van a persistir, cosa que ocurre, por ejemplo, cuando la terapia vasodilatadora es muy enérgica (68).

Por lo tanto, es importante que el estudio farmacológico experimental de agentes potencial- mente antihipertensivos, productos naturales entre ellos, incluya estudios de remodelado cardiovascular. Es relativamente sencillo identificar histológicamente si el miocardio ha sufrido el fenómeno de remodelado. Aparte de miocardiocitos hipertrofiados, la microscopía ha de mostrar la presencia de células inflamatorias, infiltrado de fibroblastos y células apoptósicas. Es conveniente que el estudio farmacológico de extractos, fracciones o compuestos aislados de origen natural, incluya este tipo de estudios para dar mayor soporte a los argumentos en su favor, como posible alternativa terapéutica para la hipertensión.

\section{Conclusiones}

En la evaluación farmacológica de productos naturales con posible actividad antihipertensiva no son incompatibles la medicina tradicional y la farmacología de síntesis. Además de plantas medicinales, el campo de productos naturales que merece ser estudiado incluye animales, microorganismos, productos marinos e insectos, como fuentes potencialmente promisorias.

Cotejando la respuesta inducida por extractos, fracciones o compuestos naturales en modelos experimentales in vivo e in vitro, entre otros tantos: la rata con hipertensión arterial inducida por deficiencia de óxido nítrico, las preparaciones de tejidos aislados como anillos vasculares y aurícula aislada, la preparación de rata sin médula y el examen histopatológico de los órganos blanco de la hipertensión, es posible estimar su potencial terapéutico, cuantificar con precisión su respuesta, hacer aproximaciones importantes sobre sus mecanismos de acción farmacológica, identificar si los compuestos activos desempeñan o no efectos sinérgicos y determinar si pueden llegar a detener la progresión de la enfermedad.

En nuestro medio sigue siendo escasa la investigación científica de productos naturales como fuente farmacológica en el campo de la hipertensión. Dada la importancia estratégica que tiene el país por su biodiversidad y el impacto nacional y mundial que aún genera la hipertensión, el estudio del potencial farmacológico de productos naturales con 
posible actividad antihipertensiva merece ser abordado con mayor profundidad.

\section{Conflicto de intereses}

Este ensayo es ajeno a conflicto de intereses del autor.

\section{Financiación}

Este ensayo no requirió financiación.

\section{Referencias}

1. Kearney PM, Whelton M, Reynolds K, Muntner P, Whelton PK, He J. Global burden of hypertension: analysis of worldwide data. Lancet. 2005;365:217-23.

2. Departamento Administrativo Nacional de Estadística. Defunciones por grupos de edad y sexo, según lista de causas agrupadas 6/67. CIE-10 de OPS. Bogotá: DANE; 2002.

3. Moser M. Are lifestyle interventions in the management of hypertension effective? How long should you wait before starting specific medical therapy? An ongoing debate. J Clin Hypertens (Greenwich). 2005;7:324-6.

4. European Society of Cardiology. 2007 Guidelines for the management of hypertension. J Hypertens. 2007;25:1105-87.

5. American Heart Association Professional Education Committee. Resistant hypertension: diagnosis, evaluation, and treatment: a scientific statement from the American Heart Association Professional Education Committee of the Council for High Blood Pressure Research. Circulation. 2008;117:e510-26.

6. Thrall G, Lip GY, Lane D. Compliance with pharmacological therapy in hypertension: can we do better, and how? J Hum Hypertens. 2004;18:595-7.

7. Newman DJ, Cragg GM, Snader KM. Natural products as sources of new drugs over the period 1981-2002. J Nat Prod. 2003;66:1022-37.

8. Butler MS. The role of natural product chemistry in drug discovery. J Nat Prod. 2004;67:2141-53.

9. Pieters L, Vlietinck AJ. Bioguided isolation of pharmacologically active plant components, still a valuable strategy for the finding of new lead compounds? J Ethnopharmacol. 2005;100:57-60.

10. Congreso de Colombia. Ley 299 de 1996. Diario Oficial No. 42845. Bogotá: Congreso de Colombia; 1996.

11. Ramírez JH, Palacios M, Gutiérrez O. Efecto diurético de la especie Salvia scutellarioides en ratas. Biomédica. 2006;26:145-9.

12. Ramírez JH, Palacios M, Gutiérrez O. Estudio del efecto antihipertensivo de la Salvia scutellarioides en un modelo de ratas hipertensas. Colomb Med. 2006;37:53-60.
13. Guerrero MF, Puebla P, Carrón R, Martín ML, Arteaga L, Román LS. Assessment of the antihypertensive and vasodilator effects of ethanolic extracts of some Colombian medicinal plants. J Ethnopharmacol. 2002;80:37-42.

14. Guerrero MF, Puebla P, Carrón R, Martín ML, Arteaga L, Román LS. Vasorelaxant effect of new neo-clerodane diterpenoids isolated from Croton schiedeanus. J Ethnopharmacol. 2004;94:185-9.

15. Onzaga IL, Rincón J, Guerrero MF. Perfil vasodilatador del extracto y la fracción flavonoide acetilada obtenida de Calea prunifolia HBK. Colomb Med. 2008;39:33-40.

16. Buitrago D, Ramos G, Rincón J, Guerrero MF. Actividad antiagregante del extracto etanólico de Solanum tuberosum en plaquetas humanas. Vitae. 2007; 14:49-54.

17. Ried K, Frank OR, Stocks NP, Fakler P, Sullivan T. Effect of garlic on blood pressure: a systematic review and meta-analysis. BMC Cardiovasc Disord. 2008;8:13.

18. Taylor JL, Rabe T, McGaw LJ, Jager AK, Van Staden J. Towards the scientific validation of traditional medicinal plants. Plant Growth Regul. 2004;34:23-37.

19. Filczewski M, Bogucka E. Reactivity of normotensive and spontaneously hypertensive rats (SHR) to some antihypertensive agents after acute and chronic treatment. Pol J Pharmacol Pharm. 1979;31:127-37.

20. Ishii $\mathbf{H}$, Itoh K, Nose T. Different antihypertensive effects of nifedipine in conscious experimental hypertensive and normotensive rats. Eur J Pharmacol. 1980;64:21-9.

21. Medina R, Cardona-Sanclemente LE, Born GV, Brown MJ. Effect of captopril and losartan on blood pressure and accumulation of LDL and fibrinogen by aortic wall and other tissues in normotensive and hypertensive rats. J Cardiovasc Pharmacol. 1997;29:125-9.

22. Levy JV. Beta-adrenergic receptor blocking drugs in spontaneous hypertension. Am J Med. 1976;61:779-89.

23. Lederballe $\boldsymbol{O}$. The experimental use of calcium antagonists in the treatment of arterial hypertension. Postgrad Med J. 1983;59(Suppl.2):84-90.

24. Xie HH, Shen FM, Zhang XF, Ji Ang YY, Su DF. Blood pressure variability, baroreflex sensitivity and organ damage in spontaneously hypertensive rats treated with various antihypertensive drugs. Eur J Pharmacol. 2006;543:77-82.

25. Antonaccio MJ, Cavaliere T, Cote D. Ontogenesis of hypertension and responsiveness to antihypertensive agents in spontaneously hypertensive rats. Blood Vessels. 1980;17:78-90.

26. Chillon JM, Capdeville-Atkinson C, Lartaud I, Guillou J, Mertès PM, Atkinson J. Chronic antihypertensive treatment with captopril plus hydrochlorothiazide improves aortic distensibility in the spontaneously hypertensive rat. Br J Pharmacol. 1992;107:710-4. 
27. Pinto YM, Paul M, Ganten D. Lessons from rat models of hypertension: from Goldblatt to genetic engineering. Cardiovasc Res. 1998;39:77-88.

28. Schiffrin EL. Endothelin and endothelin antagonists in hypertension. J Hypertens. 1998;16:1891-5.

29. Blood pressure guidelines-where are we now? Drug Ther Bull. 2008;46:65-9.

30. Ribeiro MO, Antunes E, De Nucci G, Lovisolo SM, Zatz R. Chronic inhibition of nitric oxide synthesis: a new model of arterial hypertension. Hypertension. 1992;20:298-303.

31. Massion PB, Balligand JL. Relevance of nitric oxide for myocardial remodeling. Curr Heart Fail Rep. 2007;4:18-25.

32. Zatz R, Baylis C. Chronic nitric oxide inhibition model six years on. Hypertension. 1998;32:958-64.

33. Erley CM, Rebmann S, Strobel U, Schmidt $T$, Wehrmann $M$, Osswald $H$, et al. Effects of antihypertensive therapy on blood pressure and renal function in rats with hypertension due to chronic blockade of nitric oxide synthesis. Exp Nephrol. 1995;3:293-9.

34. García-Estañ J, Ortiz MC, O’valle F, Alcaraz A, Navarro EG, Vargas $F$, et al. Effects of angiotensin-convertingenzyme inhibitors in combination with diuretics on blood pressure and renal injury in nitric oxide-deficiencyinduced hypertension in rats. Clin Sci. 2006;110:227-33.

35. Lapa AJ, Souccar C, Lima MT, Lima TC. Métodos de evaluación de la actividad farmacológica de plantas medicinales. Florianópolis, Santa Catarina: CYTED/ CNPq; 2002. p. 1-27.

36. Nichols AJ, Gellai M, Ruffolo RR Jr. Studies on the mechanism of arterial vasodilation produced by the novel antihypertensive agent, carvedilol. Fundam Clin Pharmacol. 1991;5:25-38.

37. Ito Y, Yano S, Watanabe K, Yamanaka E, Aimi N, Sakai S. Structure-activity relationship of yohimbine and its related analogs in blocking alpha-1 and alpha-2 adrenoceptors: a comparative study of cardiovascular activities. Chem Pharm Bull (Tokyo). 1990;38:1702-6.

38. Cardelus I, Bou J, Llenas J, Berga P, Gristwood RW. Cardiovascular effects of LAS 30538, a new vascular selective $\mathrm{Ca}(2+)$-channel blocker. J Pharm Pharmacol. 1992;44:830-5.

39. Lembeck F, Griesbacher T, Eckhardt M. Demonstration of extrapulmonary activity of angiotensin converting enzyme in intact tissue preparations. $\mathrm{Br} \mathrm{J}$ Pharmacol. 1990;100:49-54.

40. Lee SH, Jung YS, Lee BH, Yun SI, Yoo SE, Shin HS. Characterization of angiotensin II antagonism displayed by SK-1080, a novel nonpeptide AT1-receptor antagonist. J Cardiovasc Pharmacol. 1999;33:367-74.

41. Insel PA, Motulsky HJ. A hypothesis linking intracellular sodium, membrane receptors, and hypertension. Life Sci. 1984;34:1009-13.
42. Man in't Veld AJ, Van den Meiracker AH, Schalekamp MA. Do beta-blockers really increase peripheral vascular resistance? Review of the literature and new observations under basal conditions. Am J Hypertens. 1988;1:91-6.

43. Tallarida $\mathbf{R}$, Jacob $\mathbf{L}$. The dose-response relation in pharmacology. New York: Springer-Verlag: 1979. p. 111-41.

44. Catret M, Anselmi E, Ivorra MD, Elorriaga M, Tur R, D'ocon MP. Alpha-adrenoceptor interaction of tetrandrine and isotetrandrine in the rat: functional and binding assays. J Pharm Pharmacol. 1998;50:1267-73.

45. Spedding M, Cavero I. Calcium antagonists: a class of drugs with a bright future. Part II. Determination of basic pharmacological properties. Life Sci. 1984;35: 575-87.

46. Polster P, Christophe B, Van Damme M, Houlliche A, Chatelain P. SR 33557, a novel calcium entry blocker. I. In vitro isolated tissue studies. J Pharmacol Exp Ther. 1990;255:593-9.

47. Tsuchihashi H, Aono J, Nagatomo T, Kawada T, Ohta H, Imai S. Effects of bunitrolol on adrenergic and serotonergic receptors. Jpn J Pharmacol. 1987;45:34956.

48. Yu SM, Kuo SC. Vasorelaxant effect of isoliquiritigenin, a novel soluble guanylate cyclase activator, in rat aorta. $\mathrm{Br}$ J Pharmacol. 1995;114:587-94.

49. Spokas EG, Folco G, Quilley J, Chander P, McGiff JC. Endothelial mechanism in the vascular action of hydralazine. Hypertension. 1983;5:107-11.

50. Kaplan HR, Taylor DG, Olson SC. Quinapril: overview of preclinical data. Clin Cardiol. 1990;13:VII6-12.

51. Wong PC, Price WA Jr, Chiu AT, Duncia JV, Carini DJ, Wexler RR, et al. Nonpeptide angiotensin II receptor antagonists. XI. Pharmacology of EXP3174: an active metabolite of DuP 753, an orally active antihypertensive agent. J Pharmacol Exp Ther. 1990;255:211-7.

52. Moncada S, Palmer RM, Higgs EA. Nitric oxide: physiology, pathophysiology, and pharmacology. Pharmacol Rev. 1991;43:109-42.

53. Radomski MW, Palmer RM, Moncada S. An L-arginine/ nitric oxide pathway present in human platelets regulates aggregation. Proc Natl Acad Sci USA. 1990;87:5193-7.

54. Macallister R, Vallance P. Nitric oxide in essential and renal hypertension. J Am Soc Nephrol. 1994;5:1057-65.

55. Wilkinson IB, Franklin SS, Cockcroft JR. Nitric oxide and the regulation of large artery stiffness: from physiology to pharmacology. Hypertension. 2004;44:112-6.

56. Moncada S. Adventures in vascular biology: a tale of two mediators. Philos Trans R Soc Lond B Biol Sci. 2006;361:735-59.

57. Opie LH, Lecour S. The red wine hypothesis: from concepts to protective signalling molecules. Eur Heart J. 2007;28:1683-93. 
58. Zern TL, Fernández ML. Cardioprotective effects of dietary polyphenols. J Nutr. 2005;135:2291-4.

59. Chaudhry SI, Krumholz HM, Foody JM. Systolic hypertension in older persons. JAMA. 2004;292:107480.

60. Wright $\mathrm{Cl}$, Van-Buren L, Kroner $\mathrm{Cl}$, Koning MM. Herbal medicines as diuretics: A review of the scientific evidence. J Ethnopharmacol. 2007;114:1-31.

61. Hosohata Y, Sasaki K, Shen Y, Hattori K, Suzuki J, Nagatomo T. Bopindolol is a slowly dissociating beta 1-adrenoceptor antagonist when compared to other beta-blockers. Biol Pharm Bull. 1995;18:1066-71.

62. Yeh JL, Liou SF, Liang JC, Huang YC, Chiang LC, Wu JR, et al. Vanidipinedilol: a vanilloid-based betaadrenoceptor blocker displaying calcium entry blocking and vasorelaxant activities. J Cardiovasc Pharmacol. 2000;35:51-63.

63. Kitchen I. In vitro practical pharmacology. Oxford: Blackwell Scientific Publications; 1984. p. 33-100.

64. Voguel H. Drug discovery and evaluation, pharmacological assays. Berlin: Springer; 2002. p. 259-60.
65. Holopherne D, Mallem MY, Le Strat E, Belin de Chantemèle EJ, Gogny M, Henrion $D$, et al. CGP12177-induced haemodynamic and vascular effects in normotensive and hypertensive rats. Eur J Pharmacol. 2008;591:196-202.

66. Kawada T, Ishibashi T, Nakazawa M, Satoh S, Imai S Adrenoceptor-blocking activity and cardiohemodynamic effects of carvedilol in animals. J Cardiovasc Pharmacol. 1990;16:147-53.

67. Nasa Y, Ichihara K, Yoshida R, Abiko Y. Positive inotropic and negative chronotropic effects of (-)-cis-diltiazem in rat isolated atria. Br J Pharmacol. 1992;105:696-702.

68. Leeuv P, Kroon A. Hypertension and the development of heart failure. J Cardiovasc Pharmacol. 1998;32:S9S15.

69. Kim S, Iwao H. Molecular and cellular mechanisms of angiotensin II-mediated cardiovascular and renal disease. Pharmacol Rev. 2000;52:11-30.

70. Dominiczak AF, Devlin AM, Brosnan MJ, Anderson NH, Graham D, Clark JS, et al. Left ventricular hypertrophy and arterial blood pressure in experimental models of hypertension. Adv Exp Med Biol. 1997;432:23-33. 